\title{
Predictions of Binding of a Diverse Set of Ligands to Gelatinase-A by a Combination of Molecular Dynamics and Continuum Solvent Models
}

\author{
Tingjun Hou, Senli Guo, and Xiaojie $\mathrm{Xu}^{*}$ \\ College of Chemistry and Molecular Engineering, Peking University, Beijing 100871, P. R. China \\ Received: July 25, 2001; In Final Form: February 25, 2002
}

\begin{abstract}
The free energies of binding, $\Delta G_{\text {bind }}$, between a diverse set of eight hydroxamate inhibitors with gelatinase-A (MMP-2) were computed by using the recently developed MM/PBSA approach. In this paper, a nonbonded model was used to represent the potentials of the catalytic zinc center. Molecular dynamics (MD) simulations were used to generate the thermally averaged ensemble of conformations of the ligand-protein complexes. On the basis of the trajectories from MD simulations, the free energies of binding were calculated using molecular mechanics, the continuum solvent model, surface area estimation, and normal-mode analysis. The results show that MM/PBSA not only can rank the studied ligands effectively but also can reproduce the experimental binding free energies successfully. The predicted binding free energies correlate well with the experimental values $(r=0.84, q=0.78)$. As a comparison, the free energies of binding were also computed by using the linear interaction energy approximation (LIE). The overall agreement between the calculated and experimental values for the diverse set of ligands means that the MM/PBSA approach is a useful tool for the general evaluation of protein-ligand interactions. The analysis of the separate energy terms contributing to MM/PBSA free energy indicates that the association between hydroxamate and MMP-2 is mainly driven by more favorable van der Waals/nonpolar interactions in the complex than in solution.
\end{abstract}

\section{Introduction}

For most computational techniques in structure-based drug design, the accurate prediction of protein-ligand binding affinities with as little computational effort as possible is a key problem. Different types of approaches have been developed to explore the energy landscape of a ligand at the binding site, with different choices regarding the tradeoff between exactness and computer time. Among all these approaches, thermodynamics integration (TI) and free-energy perturbation (FEP) may be the most rigorous and strict techniques. ${ }^{1-3}$ But these two types of methods are much more difficult to apply to the problem of calculating absolute binding free energies as compared to relative ones. This difficulty is basically due to sampling and convergence problems associated with large changes in the configuration of the system. Moreover, the requirement of intensive computing time also makes TI and FEP difficult or even impossible to apply to ligand screening. In most docking procedures, some simple scoring function methods have been developed. These computationally inexpensive methods can be used to rank the ligands of large databases to find leading compounds. ${ }^{4-14}$ But the predicting accuracy of these approaches is limited because many important contributions to ligand binding such as protein flexibility, desolvation, and entropic effect are often not well-considered.

Recently, two interesting approaches-the linear interaction energy (LIE) approximation and MM/PBSA (molecular mechanics/Possion-Boltzmann surface area)-based on molecular dynamics (MD) sampling have drawn much more attention. ${ }^{15-22}$ In the LIE approach proposed by Åqvist, $\Delta G_{\mathrm{b}}$ can be computed from ligand interaction energies in the bound and free states. On the basis of the linear response consideration, only averages of the interaction energies between the ligand and its surround-

* Corresponding author. E-mail: xiaojxu@chem.pku.edu.cn. ings need to be evaluated. The binding free energies are broken down into electrostatic (Coulombic) and van der Waals contributions

$$
\begin{aligned}
\Delta G_{\text {bind }} & \approx \Delta G_{\text {bind }}^{\mathrm{el}}+\Delta G_{\text {bind }}^{\mathrm{vdw}} \\
& =\alpha\left(\langle U\rangle_{\text {bound }}^{\mathrm{el}}-\langle U\rangle_{\text {free }}^{\mathrm{el}}\right)+\beta\left(\langle U\rangle_{\text {bound }}^{\mathrm{vdw}}-\langle U\rangle_{\text {free }}^{\mathrm{vdw}}\right)
\end{aligned}
$$

where $U^{\mathrm{el}}$ and $U^{\mathrm{vw}}$ are the electrostatic and van der Waals interaction energies, respectively, between the ligand and its surroundings in protein (bound form) or in aqueous solutions (free form), \langle\rangle denotes the ensemble average over molecular dynamics or Monte Carlo simulations, and $\alpha$ and $\beta$ are empirical parameters. MD simulations were used to determine the required energy components.

The MM/PBSA technique was proposed to evaluate the solvation and binding free energies of macromolecules and their complexes. ${ }^{23}$ In this method, the average total free energy of the system, $G$, is evaluated as

$$
G=E_{\mathrm{MM}}+G_{\mathrm{PB}}+G_{\mathrm{NP}}-T S
$$

where $G_{\mathrm{PB}}$ is the polar solvation energy in the continuum solvent, which is usually computed using a finite-difference Poisson-Boltzmann (PB) model, and $G_{\mathrm{NP}}$ is the nonpolar solvation energy, which is often obtained from the solventaccessible surface area (SA). $E_{\mathrm{MM}}$ denotes the sum of the molecular mechanical (MM) energies of the molecules from internal, electrostatic, and van der Waals energies. The last term in eq 2 is the solute entropy and can be estimated by using a combination of classical statistical equations and normal-mode analysis. According to eq 2, the binding free enery of noncovalent association for a protein-ligand system can be computed as

\subsection{1/jp015516z CCC: $\$ 22.00$ (C) 2002 American Chemical Society} Published on Web 04/30/2002 


$$
\begin{aligned}
\Delta G_{\text {bind }} & =G_{\text {complex }}-G_{\text {protein }}-G_{\text {ligand }} \\
& =\Delta E_{\mathrm{MM}}+\Delta G_{\mathrm{PB}}+\Delta G_{\mathrm{NP}}-T \Delta S
\end{aligned}
$$

The thermally averaged energy terms in eq 3 are obtained from MD sampling. In practical applications, one can evaluate eq 3 by two methods: (a) run separate trajectories of complex, protein, and ligand or (b) evaluate all three terms in eq 3 using only the snapshots from a trajectory on the complex. Certainly, option (b) is 2-3 times more efficient than option (a) but uses the assumption that the free energies of the snapshots of the protein and ligand taken from the complex trajectory are comparable to those that would emerge from separate trajectories of the protein and ligand. It should be noted that approach (b) may not be the best way or the only waybut that it is merely a good approximation in some applications. ${ }^{24}$ The first application of MM/PBSA to the binding of a diverse set of ligands to a protein was reported recently by Kuhn and Kollman, ${ }^{22}$ in which the free energies of binding between nine ligands and avidin as well as between a peptide and streptavidin were computed, and the calculated values are in good agreement with the experimental ones.

Compared with most semiempirical methods used in molecular docking, the LIE and MM/PBSA techniques have several appealing features. First, these two methods are significantly faster than FEP or TI calculations because they require simulations only at the endpoints of mutations. Second, they can take into account the protein flexibility just as FEP or TI does, which is often not included in scoring function approaches. Finally, they use an explicit solvent model in their simulations; thus, the contribution of enthalpy in desolvation free energy can be reasonably handled. These advantages make these two methods potentially useful tools in structure-based ligand design. Although both of the LIE and MM/PBSA approaches are based on MD sampling, they also have obvious differences. First, LIE needs two MD simulations for ligands in the bound and free states, whereas for MM/PBSA, only one MD simulation is needed for the protein-ligand complex. Second, as for LIE, the desolvation distribution in protein-ligand association is considered implicitly in the ensemble-averaged electrostatic and van der Waals terms in eq 1 . Whereas for MM/PBSA, the desolvation energy in ligand binding is computed by using the PBSA technique. Third, for MM/PBSA, on the basis of the general empirical parameters for PB and SA calculations, we do not need a new fit for the free-energy calculations, wherease for LIE, two parameters in eq 1 may be calibrated to different protein-ligand systems.

In this paper, we used MM/PBSA to calculate binding free energies for a set of representative inhibitors interacting with gelatinase-A (MMP-2). Matrix metalloproteinases (MMPs) are key enzymes involved in connective tissue turnover in normal and pathological conditions. ${ }^{25-27}$ These zinc- and calciumdependent enzymes are synthesized as zymogens, and under physiological conditions, the proteins are selectively regulated by tissue inhibitors of metalloproteinases. The extracellular matrix functions as a medium of migration, attachment, and structural support in various cell types and tissues. Therefore, MMPs play a crucial role in matrix remodeling events of connective tissues during embryonic growth and wound healing. Among the subfamilies of MMPs, gelatinases have been considered to be very promising in drug development. Because gelatinases are thought to play an important role in triggering the processes of tumor growth, invasion, and metastasis by cleaving the vascular basement membrane that consists of type IV collagen, gelatinase inhibitors have been studied extensively

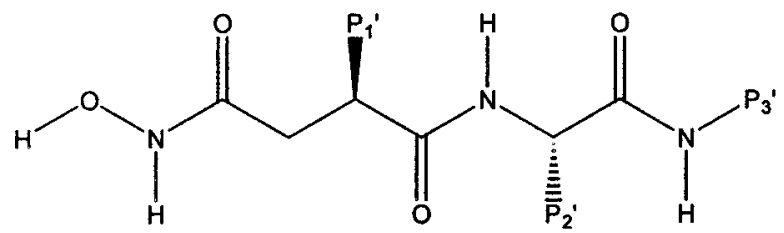

Figure 1. Common structure of the hydroxamate inhibitors.

in the search for a new type of anticancer drug. ${ }^{28,29}$ The recent resolution of the crystal structure of MMP-2 provides the opportunity to develop new drugs by the structure-based approach. $^{30}$

Many MMP inhibitors have been reported. Generally speaking, the requirement for a molecule to be an effective inhibitor of the MMPs is that it have a functional group (e.g., hydroxamic acid, carboxylic acid, and sulfhydryl, etc.) capable of attaching to the catalytic zinc atom, at least one functional group that provides a hydrogen bond interaction with the enzyme backbone, and one or more side chains that undergo effective van der Waals interactions with the enzyme subsites. Almost all ligands contain hydrophobic side chains on $\mathrm{P}_{1}{ }^{\prime}$ and $\mathrm{P}_{2}{ }^{\prime}$ substituent sites (see Figure 1), respectively. The $\mathrm{P}_{1}^{\prime}$ groups of the inhibitors are in the protein interior, whereas the $\mathrm{P}_{2}{ }^{\prime}$ group is solventexposed. Most of the effective ligands have a hydrophobic group in the $\mathrm{P}_{1}$ ' position and take advantage of the favorable van der Waals and hydrophobic interactions with the S1' pocket of MMPs. Hydrophilic interactions such as hydrogen bonds between the ligands and receptor also significantly contribute to ligand binding. From experimental results, the ranking of the potency of the inhibitors according to the functional group binding to the catalytic zinc center is hydroxamate $>$ sulfhydryl $>$ phosphinate $>$ aminocarboxylate $>$ carboxylate. ${ }^{31}$ Many of the currently designed inhibitors for the MMPs are based on hydroxamate zinc-binding moieties coupled to a peptidic framework. So in this paper, a set of representative MMP-2 inhibitors - hydroxamates with wide range of binding affinitieshave been selected for free-energy calculations. ${ }^{32-34}$ As seen in Figure 2, the eight ligands we studied are considerably different in structure and size. Before our work, several papers concerned only with the application of MM/PBSA to the binding of a diverse set of ligands to protein were reported. ${ }^{21,22}$ The validity of MM/PBSA to rank protein-ligand systems should be carefully investigated, so the first aim of this paper is to evaluate the accuracy of the binding affinities obtained with $\mathrm{MM} / \mathrm{PBSA}$ and to determine if the results from MM/PBSA are accurate enough to allow for the discovery of lead compounds. In this work, the LIE technique is also used to estimate the binding affinities for the studied protein-ligand systems in a comparative fashion.

\section{Methods}

2.1. General. All of the MD simulations were done with the AMBER 6.0 molecular simulation package. An AMBER force field was used for molecular minimizations and dynamics. ${ }^{35}$ The analysis of MD trajectories was performed using AMBER 6.0 and in-house software. Quantum mechanical calculations were carried out using Mopac 7.0 $0^{36}$ and Gaussian 98. ${ }^{37}$ All the calculations were performed on a 2-CPU SGI Octane workstation and a homemade Linux parallel computing system with 22 Pentium PIII733 CPUs. Visualization and the other molecular modeling were performed using the Insight II $^{38}$ and Sybyl 6.7 molecular simulation package. ${ }^{39}$

2.2. Construction of the Initial Structures. A recent report described the structure of the full-length proform of MMP-2 
<smiles>CC(C)CC(CC(=O)N(C)O)C(=O)N(N)[C@H](CC1CCCCC1)C(=O)N(C)CCc1ccccc1</smiles>

(a1)<smiles>CN(CCc1ccccc1)C(=O)[C@H](CC1CCCCC1)N(N)C(=O)[C@H](CCc1ccccc1)CC(=O)N(C)O[Na]</smiles>

(a3)<smiles>CON(C)C(=O)C[C@@H](CCCC1CCCCC1)C(=O)N(C)[C@@H](CC1CCCCC1)C(=O)N(C)CCc1ccccc1</smiles>

(a5)<smiles>Cn1c(=O)n(C[C@H](C(=O)NO)[C@H](CC2CCCC2)C(=O)N2CCCCC2)c(=O)n1C</smiles>

(a7)

Figure 2. 2D structures of the hydroxamate inhibitors.

(MMP-2, PDB code: 1QIB). ${ }^{30}$ But the complex structures of MMP-2 and its inhibitors are unavailable, so it is a challenge for us to construct the initial structures for MD simulations. The catalytic domain of the MMP-2 is similar to that of MMP3 . The same residues form the substrate binding pockets, and coordination of the catalytic $\mathrm{Zn}^{2+}$ ion is quite similar. Also, the binding site is identical to a well-conserved motif found in all known MMP structures. According to the literature, the basic structures of MMP inhibitors are quite similar; moreover, considerable insight into MMP ligand interaction has revealed that the inhibitors of different MMPs generally adopt similar binding modes with their receptors. Therefore, we believe that the constructed complexes of MMP-2 with hydroxamates from<smiles>[1H]N(O[Na])C(=O)C[C@@H](Cc1ccccc1)C(=O)N(C)[C@H](CC1CCCCC1)C(=O)N(C)CCc1ccccc1</smiles>

(a2)<smiles>[2H]N(O[13CH3])C(=O)C[C@@H](CCCc1ccccc1)C(=O)N(C)[C@H](CC1CCCCC1)C(=O)N(C)CCc1ccccc1</smiles>

(a4)<smiles>[1H]N(O)C(=O)C[C@@H](CCCc1ccc(Cl)cc1)C(=O)N(N)[C@@H](CC1CCCCC1)C(=O)N(C)CCC(=O)O</smiles>

(a6)<smiles>N[C@@H](C(=O)NO)[C@H](CC1CCCC1)C(=O)N1CCN(Cc2ccc3c(c2)OCO3)CC1</smiles>

(a8) the crystal structure of an MMP-3 complex are precise enough to be the initial structures for MD simulations. In this paper, an X-ray crystal of MMP-3 with a hydroxamate inhibitor (PDB code: 1BIW) obtained by Natchus et al. was used as the template molecule. ${ }^{40}$

In the current work, we selected eight hydroxamate inhibitors from the literature, and some of them show a strong binding affinity to MMP-2. ${ }^{32-34}$ The compounds studied are shown in Figure 2. The construction of the complex structure of MMP-2 with the studied molecules was divided into three stages. First, the structural alignment was used to superimpose MMP-2 onto MMP-3. Then, the hydroxamate inhibitor was extracted from $1 \mathrm{BIW}$ and merged into 1QIB. Finally, the structure of the 
hydroxamate inhibitor was modified to obtain the studied molecules. During this model-building process, the conformation of the protein was not altered, and the structures of the inhibitors were altered minimally to avoid unacceptable atom bumps.

2.3. Force Field. The van der Waals nonbonded parameters for the zinc center were taken from Hoops et al. ${ }^{41}$ The zinc and calcium ions were assigned the full formal charge of $2|\mathrm{e}|$. The calcium ion VDW radius was taken from the Åqvist parameter set. ${ }^{42}$ Some missing parameters concerned with inhibitors were obtained from the newest AMBER force field (parm99) that was revised by Wang et al. ${ }^{43,44}$

All the molecules shown in Figure 2 that were modified from the hydroxamate in 1BIW were fully minimized by the AM1 Hamiltonian in MOPAC 7.0. The Hartree-Fock method with the 6-31G* level used in the Gaussian 98 program were used to determine electrostatic potentials. ${ }^{45}$ The RESP fitting technique in AMBER was applied to determine the partial charges. ${ }^{46}$

2.4. Molecular Dynamics. All MD simulations were carried out at $300 \mathrm{~K}$, with the ligand bound to the protein and with a cap of waters around the complex filled up to $20 \AA$ from the center of mass of the ligand. The explicit solvent model TIP3P was used for water. ${ }^{47}$ The SHAKE procedure was employed to constrain all bonds involving at least one hydrogen atom. The time step of the simulations was 2.0 fs with a cutoff of $12 \AA$ for the nonbonded interactions. The nonbonded pairs were updated every 30 steps. Prior to the MD simulations, the system was minimized with harmonic position constraints for all protein heavy atoms. The constraints were 5000, 1000, 100, and 10 $\mathrm{kcal} / \mathrm{mol} / \AA^{2}$. Subsequently, a cycle of minimization was done to relax all the atoms without constraints. The maximum minimization steps were 10000 , and the convergence criterion for the energy gradient was $0.5 \mathrm{kcal} / \mathrm{mol} / \AA^{2}$. In MD simulations of bound ligands in protein, all residues within $16 \AA$ were allowed to move, whereas the other atoms were restrained by a $15 \mathrm{kcal} / \mathrm{mol} / \AA^{2}$ harmonic force. MD simulation procedures for the protein-ligand systems involved (1) 100 ps of MD equilibration and (2) $200 \mathrm{ps}$ of MD simulations for data collection. In the data-collection stage, the snapshot was recorded every $500 \mathrm{fs}$ in the trajectory file.

To determine the parameters of the LIE model (eq 1), MD simulations were also performed for the unbound ligand in a 20 - $\AA$ sphere of water. In MD simulations of an unbound ligand in aqueous solution, a position constraint for the ligand's heavy atom that is closest to the center of mass of the ligand was applied. For the unbound ligands in the water cap, after $100 \mathrm{ps}$ of MD equilibration, 100 ps of MD simulations was performed for data collection.

2.5. MM/PBSA Calculations. The energy terms in eq 3 were calculated separately. The gas-phase molecular mechanical (MM) energy was averaged over all the snapshots. All MM calculations were performed using the anal module in AMBER with a nonbonded cutoff of $99 \AA$ and a dielectric constant of 1 in the absence of any solvent.

The electrostatic contribution to the solvation energy, $\Delta G_{\mathrm{PB}}$, was calculated using the DelphiII software package, ${ }^{48}$ which solves the Poisson-Boltzmann equation numerically and calculates the electrostatic energy according to the electrostatic potential. The grid size was defined as $0.5 \AA$. The radius of the probe molecule was set to $1.4 \AA$. The charges used in the PB calculations were taken from the AMBER parameter set (protein) and the RESP fittings (ligand). The radii of atoms were taken from the PARSE parameter set. ${ }^{49}$ The radii for $\mathrm{Ca}^{2+}$ and $\mathrm{Zn}^{2+}$, which were absent from the PARSE set, were defined as 1.97 and $1.4 \AA$ A, respectively. ${ }^{21}$ The iterative Delphi calculations were run for 1000 steps. The solvent-accessible surface (SAS) was calculated using the MSMS program. ${ }^{50}$ The nonpolar contribution to the desolvation free energy was calculated as $0.00542 \times \mathrm{SAS}+0.92 \mathrm{kcal} / \mathrm{mol}$.

2.6. Nmode Analysis. In the MM/PBSA calculation, no solute entropy contribution was considered. We estimated the conformational entropy contributions (translation, rotation, and vibration) to the binding free energy using normal-mode analysis, which was carried out using the nmode program in AMBER 6.0. We must point out that the nmode calculations were an $N^{3}$ problem, where $N$ is the number of atoms in question, so it is very time-consuming. To simplify the calculations, the residues within an 8 - $\AA$ sphere around the ligand were cut out from an MD snapshot for each ligand-protein complex. The open valences were saturated by adding hydrogen atoms in Sybyl. The corresponding uncomplexed reactants were generated by removing the atoms of the protein and the atoms from the reduced complex structure. Then, each of the structures was fully minimized using conjugate gradient strategy for 50000 steps in the presence of a distance-dependent dielectric $(\epsilon=$ $4 r_{i j}$ ) using the sander program in AMBER. Consequently, the structures were further minimized with the Newton-Raphson technique for 500 steps using the nmode program. Last, the entropy for each structure was calculated using classical statistical formulas and normal-mode analysis. To reduce the statistical error, a snapshot was extracted and analyzed every 20 ps. The final entropy estimate was averaged over 10 snapshots.

2.7. Construction of the LIE Models. The average electrostatic and van der Waals interaction energies between the inhibitors (bound and free) and their respective environments were determined from the MD simulations. Then, a genetic algorithm (GA) was used to fit the present energetic components to the experimental $\Delta G_{\mathrm{b}}$ values. ${ }^{51-53}$ During GA optimizations, the multiple linear regression coefficient $(r)$ was defined as the fitness score to evaluate the LIE models. The reliabilities of the models were tested by the leave-one-out cross-validation technique.

\section{Results and Discussion}

To obtain the reliable, thermally averaged energy terms in eq 3, the length of the MD simulations should be sufficiently long. In principle, the longer the simulation is performed, the more reliable are the thermally averaged properties that are obtained. In practice, a relatively short simulation seems adequately accurate in this case. Figure 3 shows the time evolution of the averaged, nonbonded van der Waals and electrostatic interactions between $\mathbf{a} 1$ and MMP-2 during the MD simulations. It seems that these two energy terms fluctuate within relatively small regions. Table 1 shows the averaged, nonbonded interactions between $\mathbf{a} \mathbf{1}$ and MMP-2 using a different time interval. The mean energy difference between the first 100 and $200 \mathrm{ps}$ is $0.01 \mathrm{kcal} / \mathrm{mol}$ for the electrostatic energy and $0.40 \mathrm{kcal} / \mathrm{mol}$ for the van der Waals energy. In the cases we studied, we observed that the mean energy converged within the 100-ps simulation, so the mean energy from the 200-ps conformational sampling is reliable enough.

3.1. Coordinating Form of the Zinc Center. Here, it must be noted that the ligands studied are coordinated with a zinc center, which is a challenge for molecular modeling. We face the following choice for the potential model of the zinc atom: the nonbonded or bonded model. In the nonbonded approach, nonbonded electrostatic and van der Waals terms are used to model the metal-ligand/enzyme interactions. In the bonded 


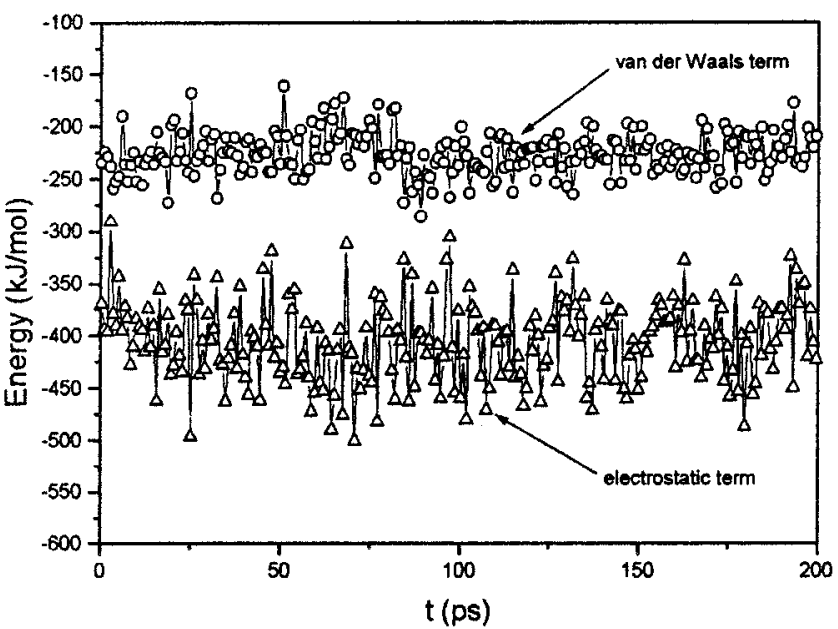

Figure 3. Fluctuations of the nonbonded interactions during MD sampling.

TABLE 1: Averaged Nonbonded Interactions between a1 and MMP-2 Using Different Time Intervals

\begin{tabular}{ccc}
\hline$t(\mathrm{ps})$ & $E_{\text {ele }}(\mathrm{kcal} / \mathrm{mol})$ & $E_{\text {vdw }}(\mathrm{kcal} / \mathrm{mol})$ \\
\hline $0-50$ & $-53.95(4.32)$ & $-96.87(8.86)$ \\
$0-100$ & $-54.22(5.02)$ & $-97.27(9.14)$ \\
$0-150$ & $-54.33(4.89)$ & $-97.37(8.98)$ \\
$0-200$ & $-54.23(4.74)$ & $-96.87(8.88)$
\end{tabular}

approach, the coordinate bonds between the ions and the ligand/ enzyme are described by the usual terms including bond stretching, angle bending, and torsional terms. Obviously, neither of these two models is entirely satisfactory. The nonbonded approach is simple, but it is very sensitive to the van der Waals parameters of the atoms that are concerned with the coordinate bonds and can suffer from the inability to retain low coordination numbers. The bonded approach is more potentially complete and the coordination number can be well-maintained during the whole MD run, but by using the bonded model, the coordinate center is almost frozen. If we give an improper coordinating form of the coordination center in the initial model, the imposed problem will be preserved. In several previous papers about molecular modeling of metalloproteins, ${ }^{41,54}$ the use of the bonded model of the zinc center produces good results. In Donini and Kollman's previous work on free-energy calculations between several carboxylate ligands with MMP- $1,{ }^{21}$ the nonbonded zinc model is used, and the calculated results seem acceptable. In the current work, the nonbonded zinc model is adopted on the basis of two considerations. First, using the bonded model, the $\Delta E_{\mathrm{MM}}$ term in eq 3 is sensitive to the proper parametrization of the zinc center. Until now, the force field parameters from the previous work have not been complete enough to represent all bonded terms between the zinc center and the hydroxamate group. ${ }^{41}$ Second, in previous work on the prediction of the free energy of bindings between 15 hydroxamates and MMP-2, a bonded zinc center was used. ${ }^{55}$ Therefore, in this paper, the use of the nonboned model can be directly compared with the use of the bonded model.

During the construction of the initial models for MD simulations, the crystal structure of an MMP-3 complex (1BIW) was treated as the template. In the crystal structure of the MMP-3 complex with hydroxamate inhibitor, the catalytic zinc is pentacoordinated ( 3 His and 2 oxygens from the inhibitor zinc binding group; see Figure 4), so in the initial models that were constructed of MMP-2 complexed with hydroxamates, the catalytic zinc is also pentacoordinated.

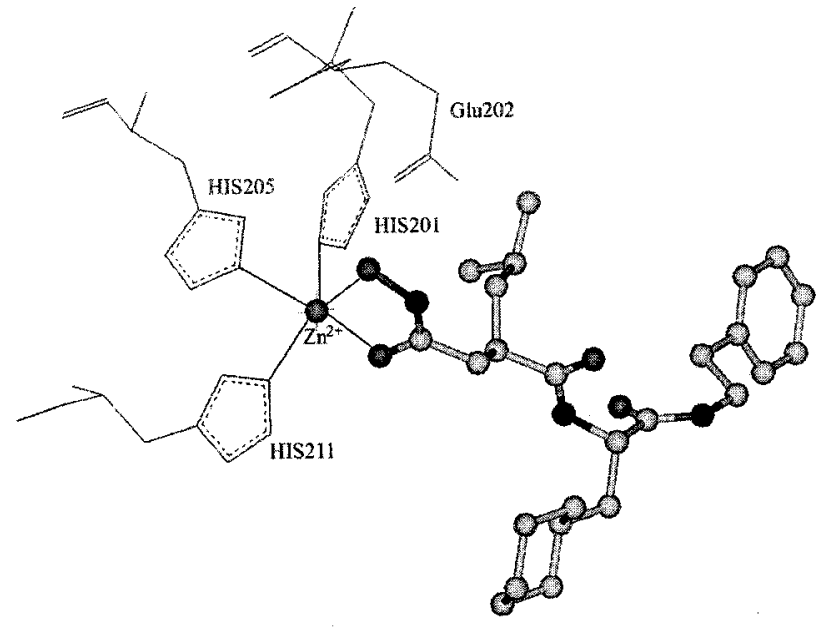

(a)

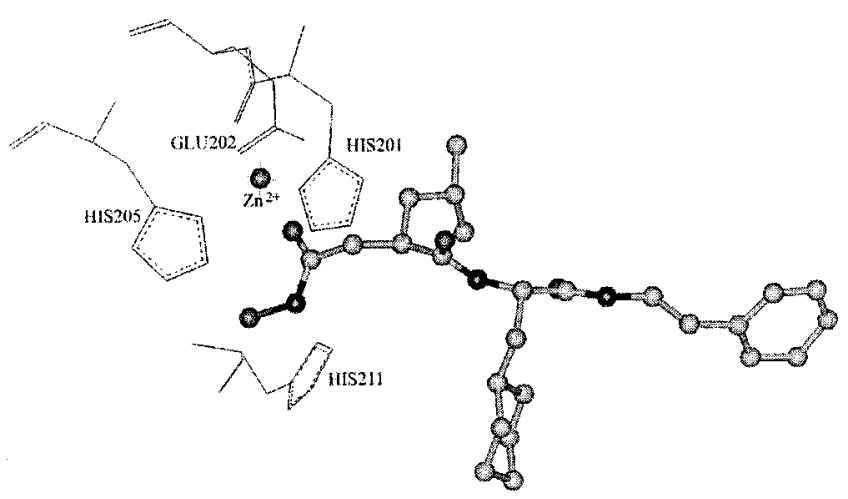

(b)

Figure 4. Coordination of the catalytic zinc in (a) the initial conformation and (b) the conformation after 200-ps MD simulations. The ligand $\mathbf{a} 1$ is shown as a ball-and-stick diagram.

The MD trajectories show that after equilibration the change in the coordinate form of the catalytic zinc is obvious. Figure 4 shows the structures of the catalytic zinc, the three ligand histidines, and the inhibtor a1 after 200-ps MD simulations. In Figure $4 \mathrm{~b}$, the catalytic zinc is also pentacoordinated, but the ligand groups are different from those in Figure 4a. The nitrogen atoms in His201 and His205 can still form stable coordinate bonds with the catalytic zinc, and the distances between those two nitrogens and the zinc atom are 2.02 and $2.01 \AA$, respectively. The distance between the nitrogen atom in His 211 and the zinc atom is $5.85 \AA$, which is obviously beyond the distance in which it is possible to form stable coordinate bonds. In Figure 4b, the two oxygen atoms in Glu202 come within 1.75 and $1.85 \AA$ of the zinc, which may produce stable coordinate bonds with the catalytic zinc. In our previous work, when adopting the bonded zinc model, the coordinate form of the zinc atom was well-preserved. ${ }^{55}$ In the current work, when adopting the nonbonded model, the catalytic zinc is also pentacoordinated, but the coordinate form is different from that in the initial model. Nevertheless, the coordinate form from our MD simulations should be further validated by experiments.

3.2. LIE Models. The differences between the average electrostatic and van der Waals interaction energies between the inhibitors (bound and free) and their respective environments were determined during the MD simulations (see Table 2).

In the initial two-term model proposed by Aqvist (eq 1), the parameter for the electrostatic part was fixed $(\alpha=0.5)$, and $\beta$ was calibrated according to the system. ${ }^{56,57}$ In Table 3, the LIE expression (eq 1$)$ and $\alpha(0.50)$ and $\beta(0.39)$ were ineffective at 
TABLE 2: Differences between the Average Electrostatic and van der Waals Interaction Energies between the Inhibitors (Bound and Free) and Their Respective Environments from MD Sampling

\begin{tabular}{ccc}
\hline no. & $\left\langle U_{\text {vdw }}\right\rangle(\mathrm{kcal} / \mathrm{mol})$ & $\left\langle U_{\text {elec }}\right\rangle(\mathrm{kcal} / \mathrm{mol})$ \\
\hline $\mathbf{a 1}$ & -12.53 & -38.54 \\
$\mathbf{a} 2$ & -11.13 & -13.14 \\
$\mathbf{a 3}$ & -21.41 & -21.89 \\
$\mathbf{a}$ & -24.00 & -24.71 \\
$\mathbf{a 5}$ & -13.93 & -27.58 \\
$\mathbf{a 6}$ & -29.02 & -16.53 \\
$\mathbf{a} 7$ & -10.66 & -20.02 \\
$\mathbf{a 8}$ & -23.49 & 21.84
\end{tabular}

TABLE 3: Experimental and Calculated $\Delta G_{\mathrm{b}}$ Values Using Different Fitted Models

\begin{tabular}{lrrrr}
\hline & \multicolumn{4}{c}{$\Delta G_{\mathrm{b}}(\mathrm{kcal} / \mathrm{mol})$} \\
\cline { 2 - 5 } & & & & model 3 \\
& & $\alpha=0.5$, & $\alpha=0.13$, & $\beta=0.09$, \\
no. & expt. & $\beta=0.39$ & $\beta=0.49$ & $\gamma=-17.18$ \\
\hline $\mathbf{a 1}$ & -12.93 & 2.47 & -11.19 & -11.54 \\
$\mathbf{a} 2$ & -7.80 & -1.78 & -7.17 & -8.85 \\
$\mathbf{a 3}$ & -11.80 & -0.33 & -13.35 & -12.94 \\
$\mathbf{a 4}$ & -13.92 & -0.61 & -14.98 & -14.02 \\
$\mathbf{a 5}$ & -11.13 & 1.04 & -10.43 & -11.03 \\
$\mathbf{a 6}$ & -15.01 & -2.63 & -16.36 & -14.92 \\
$\mathbf{a 7}$ & -9.28 & 0.28 & -7.84 & -9.31 \\
$\mathbf{a 8}$ & -10.49 & 0.17 & -8.62 & -9.75 \\
$r^{2}$ & & 0.01 & 0.88 & 0.88 \\
$q^{2}$ & & -26.16 & 0.57 & 0.83 \\
$F$ & & 0 & 32.80 & 17.95
\end{tabular}

producing the experimental $\Delta G_{\mathrm{b}}$ values for inhibitors $\mathbf{a 1}-\mathbf{a 8}$ using the energetic results from the present simulations. Then, another two-term equation in which the values of $\alpha$ and $\beta$ were allowed to be optimized was reparametrized. In this model, $\beta$ is equal to 0.49 , and $\alpha$ is only 0.13 , which is greatly different from the value that is usually used (0.5) (see model 2 in Table $3)$. The new fit resulted in substantial improvement $\left(r^{2}=0.88\right.$, $\left.q^{2}=0.57\right)$.

In previous work on the binding free-energy calculations of 15 hydroxamate inhibitors with gelatinase-A, ${ }^{55}$ several linear models consisting of different energy components were tested. We found that besides the Coulombic and van der Waals energy terms that are normally used the introduction of a constant term could significantly improve the correlation. In the current work, a three-parameter model with a constant term was also attempted (see equation below).

$$
\Delta G_{\text {bind }}=\alpha\left(\Delta U_{\text {elec }}\right)+\beta\left(\Delta U_{\text {vdw }}\right)+\delta
$$

The new fit resulted in significant improvement, which is indicated by the high $q^{2}(0.83)$ value. Three parameters for eq 1 were $\alpha=0.09, \beta=0.32$, and $\delta=-17.18$. The average unsigned error for this fit with eight inhibitors and three parameters is $0.58 \mathrm{kcal} / \mathrm{mol}$, so model 3 in Table 3 was determined as the best statistical model. The constant $\delta$ yields an attractive term of $-17.18 \mathrm{kcal} / \mathrm{mol}$. In our previous work using the bonded model, we obtained three parameters for eq 4: $\alpha=0.191, \beta=0.827$, and $\delta=-7.405$, respectively. ${ }^{55} \mathrm{We}$ think that regardless of whether the nonbonded zinc model or the bonded model is used all the ligands in question adopt a similar coordinate form with $\mathrm{Zn}$ using the same functional group. Therefore, the systematic errors in the energetic or conformational assessment of the interaction between zinc and the ligand should be constant, which may be absorbed in the constant term in eq 4.
3.3. MM/PBSA Models. The free energies of binding using MM/PBSA and normal-mode analysis are shown in Table 4 and Figure 5. In Figure 5, the experimental $\Delta G_{\text {bind }}$ values shows good correlation with the calculated values, which is indicated by the high values of the coefficients of linear regression $(r=$ $0.84, q=0.78)$. The average absolute error in the regression line $(y=1.68 x+9.98)$ is $2.9 \mathrm{kcal} / \mathrm{mol}$, which is better than the error reported by Kuhn and Kollman. In Kuhn's work with nine inhibitors of avidine and streptavidin, the correlation between the calculated and experimental $\Delta G_{\mathrm{b}}$ values is quite good $\left(r^{2}=0.92\right)$, but the obtained average error of $3.3 \mathrm{kcal} /$ mol is relatively large. ${ }^{22}$ The values in Table 4 show that despite the overestimation of $4.8 \mathrm{kcal} / \mathrm{mol}$ for ligand $\mathbf{a} 2$ and $7.4 \mathrm{kcal} /$ mol for $\mathbf{a} 7$ the overall agreement between the calculated and experimental values for this diverse set of ligands is quite good. Moreover, we find that besides ligand $\mathbf{a} 4$, the calculated $\Delta \Delta G$ values of the other ligands are positive. Our calculations are consistent with those reported by Donini et al. ${ }^{21}$ When binding involves divalent ions such as $\mathrm{Zn}^{2+}$ and $\mathrm{Mg}^{2+}$, continuum models will overestimate the desolvation penalty of moving a divalent ion from a high to a low dielectric medium. ${ }^{25}$

Further insight into the forces involved in substrate binding can be obtained by analyzing the MM/PBSA free-energy contributions, which are listed in Table 4 for the eight ligands. Comparing the van der Waals/nonpolar $\left(\Delta E_{\mathrm{vdw}}+\Delta G_{\mathrm{SA}}\right)$ contributions with the electrostatic $\left(\Delta E_{\mathrm{es}}+\Delta G_{\mathrm{PB}}\right)$ contributions, we find that the association between hydroxamate and MMP-2 is mainly driven by more favorable van der Waals/nonpolar interaction in the complex than in solution. The electrostatic interactions between MMP-2 and hydroxamates are quite strong, but the electrostatic interactions between the solvent (water molecules) and the ligand are much stronger. Thus, when a ligand transfers from the solvent to the binding pocket, the electrostatic contributions for hydroxamates are unfavorable to ligand binding. After careful observations, it is very interesting to find that the absolute free energies of binding are closely correlated with the van der Waals/nonpolar contribution (Figure 6 ). The structural analysis demonstrates that the hydrophobic group in the $\mathrm{P}_{1}$ ' position takes advantage of the favorable van der Waals and hydrophobic interactions with the S1' pocket of MMPs. In the S1' pocket, four residues including Leu 197, Val 198, Leu 218, and Tyr 223 constitute a relatively large hydrophobic core, which can generate strong van der Waals and hydrophobic interactions with the inhibitors. For example, the hydroxamates $\mathbf{2} 2-\mathbf{a} 4$ show a clear stepwise increase in MMP-2 binding affinities according to the straight elongation of the $\mathrm{P}_{1}$ ' group. Among ligands $\mathbf{a} 2, \mathbf{a} 3$, and $\mathbf{a} 4$, the $\Delta E_{\mathrm{vdw}}+$ $\Delta G_{\mathrm{SA}}$ value of the ligand $\mathbf{a} 2$ is the weakest because the short $\mathrm{P}_{1}^{\prime}$ group cannot produce an effective surface that is complementary to the $S_{1}{ }^{\prime}$ pocket. In viewing the structure for the a2 complex, it is obvious that the nonpolar $\mathrm{P}_{1}{ }^{\prime}$ group is in close contact only with the hydrophobic side chains of Val 198 and Tyr 223. Whereas the $\mathrm{P}_{1}{ }^{\prime}$ of $\mathbf{a} 2$ is elongated by one $\mathrm{CH}_{2}$ to $\left(\mathrm{CH}_{2}\right)_{2} \mathrm{Ph}$, the binding free energy of $\mathbf{a} 3$ increases greatly. The key contributions to the 1000-fold increase in $K_{\mathrm{i}}$ of $\mathbf{a} 3$ compared to that for $\mathbf{a} 2$ are obvious. The longer $\mathrm{P}_{1}{ }^{\prime}$ group can be located in the deeper interior of the S1' pocket, which can produce effective surface contacts with not only the nonpolar side chains of Val 198 and Tyr 223 but also those of Leu 197 and Leu 218. As a result, $\mathbf{a} 3$ can produce more favorable van der Waals interactions $(-47.7 \mathrm{kcal} / \mathrm{mol})$ with its environments (including protein and solvent) than can a2 $(-28.5 \mathrm{kcal} / \mathrm{mol}) \mathrm{o}$. The structure for the complex of a4 shows that the $\mathrm{P}_{1}{ }^{\prime}$ group of $\mathbf{a} 4$ can form better surface contacts with the $S_{1}$ ' pocket, which leads 
TABLE 4: Energy Contributions to the Free Energy of Binding, $\Delta G_{\text {bind, }}$, between MMP-2 and the Hydroxamate Inhibitors

\begin{tabular}{|c|c|c|c|c|c|c|c|}
\hline no. & $\Delta E_{\mathrm{es}}$ & $\Delta E_{\mathrm{vdw}}$ & $\Delta G_{\mathrm{SA}}$ & $\Delta G_{\mathrm{PB}}$ & $-T \Delta S$ & $\Delta G_{\text {bind }}$ & $\Delta \Delta G$ \\
\hline a1 & $-75.5 \pm 5.8$ & $-38.3 \pm 3.7$ & $-5.7 \pm 0.2$ & $85.8 \pm 4.9$ & 17.7 & -12.0 & 0.9 \\
\hline a2 & $-54.5 \pm 4.9$ & $-28.5 \pm 3.2$ & $-5.0 \pm 0.1$ & $71.7 \pm 2.8$ & 15.9 & -0.40 & 7.4 \\
\hline a3 & $-68.7 \pm 6.1$ & $-47.7 \pm 3.5$ & $-6.3 \pm 0.1$ & $89.9 \pm 4.2$ & 18.2 & -10.6 & 1.2 \\
\hline a4 & $-80.3 \pm 6.4$ & $-55.0 \pm 4.1$ & $-6.8 \pm 0.1$ & $106.5 \pm 4.0$ & 19.3 & -12.3 & 1.6 \\
\hline a5 & $-66.1 \pm 4.9$ & $-44.0 \pm 4.0$ & $-6.2 \pm 0.2$ & $82.1 \pm 4.2$ & 19.9 & -14.3 & -3.2 \\
\hline a6 & $-72.0 \pm 6.2$ & $-58.2 \pm 4.2$ & $-6.9 \pm 0.1$ & $100.1 \pm 3.9$ & 18.6 & -13.1 & 1.9 \\
\hline a7 & $-42.4 \pm 5.8$ & $-35.6 \pm 3.7$ & $-5.2 \pm 0.2$ & $70.9 \pm 4.7$ & 9.7 & -4.5 & 4.8 \\
\hline a8 & $-1.9 \pm 3.6$ & $-48.4 \pm 2.4$ & $-5.3 \pm 0.1$ & $46.2 \pm 3.3$ & 9.9 & -8.4 & 2.1 \\
\hline
\end{tabular}

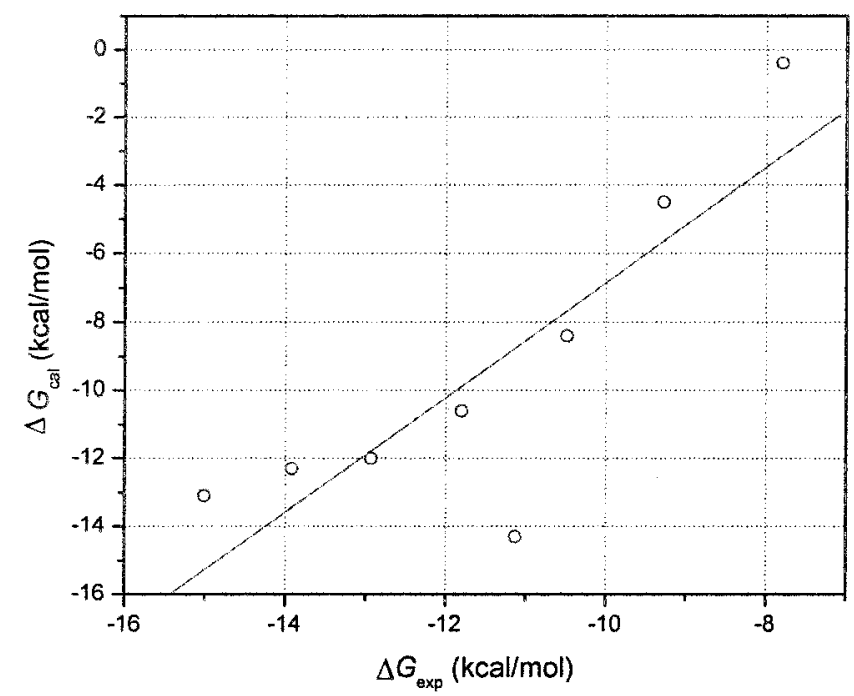

Figure 5. Correlation between the predicted binding free energies and the experimental values.

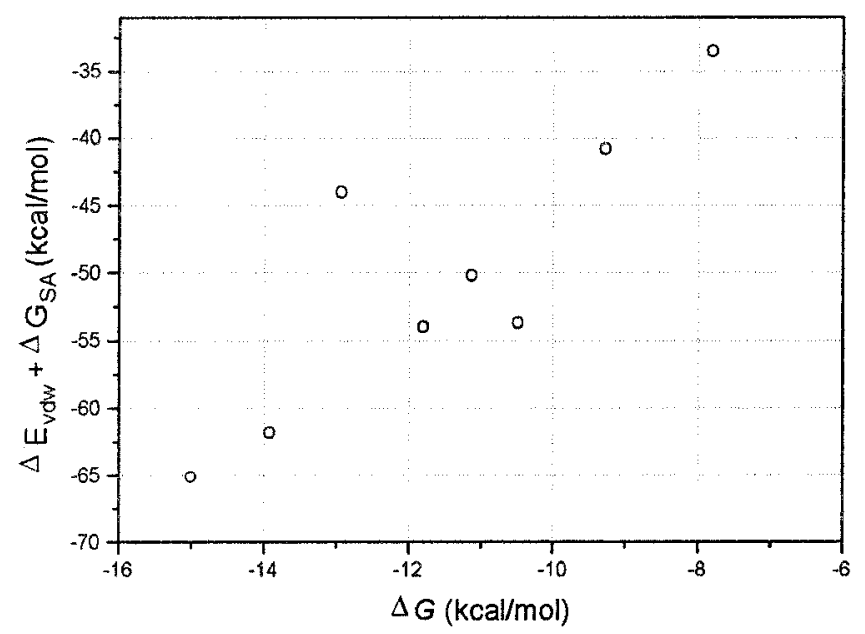

Figure 6. Relationship between the free energy of binding, $\Delta G_{\text {bind, }}$ and the van der Waals and nonpolar contributions to solvation.

to a large reduction of van der Waals interactions $(-55.0 \mathrm{kcal} /$ mol). Moreover, the MD trajectories for a4 show that the benzene rings of the inhibitors and Tyr 223 prefer to be parallel and that the pair of parallel benzene rings may produce relatively strong aromatic stacking interactions. The stronger van der Waals interaction of a4 leads to a further 36-fold increase in binding relative to that of $\mathbf{a} 3$.

It is particularly encouraging for the MM/PBSA approach that the large differences of $\Delta E_{\mathrm{es}}$ or $\Delta E_{\mathrm{vdw}}$ among ligands can be compensated by the other contributions, resulting in only slight deviations from experiment. If we consider only the $\Delta E_{\text {ele }}$ and $\Delta E_{\mathrm{vdw}}$ energy terms, the differences in the binding affinities between two ligands should be overestimated. For example, the difference in the $\left(\Delta E_{\text {ele }}+\Delta E_{\mathrm{vdw}}\right)$ value between $\mathbf{a} \mathbf{3}$ and $\mathbf{a} 4$ is $18.91 \mathrm{kcal} / \mathrm{mol}$, whereas that of the binding affinities is only
$2.12 \mathrm{kcal} / \mathrm{mol}$. If we consider the difference between $\Delta G_{\mathrm{PB}}$ in desolvation, the discrepancy can be effectively compensated, so the hydration ability for the unbound inhibitor is also very important.

3.4. Comparison between LIE and MM/PBSA. Considering only the unsigned absolute errors between the calculated and experimental free energies, we find that the predictions obtained with LIE are much better than those obtained with MM/PBSA. Undoubtedly, the quality of these two methods cannot be evaluated only by the statistical significance of the linear regression between the calculated and experimental binding free energies. LIE calculations provide good results, but the best LIE model includes three empirical parameters. In the fitting process of LIE calculations, some random or systematic errors could be reduced. In previous work, many LIE models have been proposed by considering different protein-ligand systems. ${ }^{19-21,55,58}$ In a specific system, the LIE mode can produce very good prediction, but unfortunately, the LIE models derived from other work contains different empirical parameters. In the initial work of Aqvist et al., $\beta \approx 0.16$ was derived to give the best fit to the experimental binding data, and the electrostatic scaling factor, $\alpha=0.5$, follows from the quadratic dependence of the free energy on solute charge, as embodied in the Born model for ion solvation. ${ }^{19}$ The calculations of Paulsen and Ornstein show that $\alpha \approx 0.5$ and $\beta \approx 1.043$ are the best values to use to correlate the calculated binding free energies with the respective experimental values. ${ }^{20}$ In Wang's work, $\alpha \approx 0.5$ and $\beta \approx 1.0$ can give the best predicted binding affinities to a group of avidin ligands using the AMBER force field. ${ }^{21}$ Jones-Herzog and Jorgensen observed that the addition of another term concerned with molecular solvent-accessible surfaces as well as $\alpha=0.131$ and $\beta=0.131$ could produce the best correlation. In our previous work, we found that $\alpha \approx 0.19$ and $\beta \approx 0.83$ as well as a constant term gives the best prediction of the binding free energies. ${ }^{55}$ All of these models seem quite different from the LIE model discussed in this paper. The high reliability of the empirical parameters of LIE from the fitting makes this method very difficult to apply to the discovery of lead compounds. If we cannot effectively represent the relationships between $\Delta G_{\text {bind }}$ and $\left\langle U_{\mathrm{vdw}}\right\rangle$ and $\left\langle U_{\mathrm{es}}\right\rangle$ according to different protein-ligand systems, the LIE technique is only a QSARlike study, and its applications may be greatly limited.

It is particularly encouraging to discover that if we have reliable empirical parameters related to PBSA calculations we do not need any new fit for different protein-ligand systems; therefore, MM/PBSA may be an attractive tool for this applications in different protein-ligand systems.

For MM/PBSA, the calculation of the change in solute entropy upon complexation may deserve the most attention. In the current work, normal-mode analysis was used to compute the entropic contribution in ligand binding. The normal-mode analysis is based on the harmonic approximation; there is also likely to be a significant systematic error that is not included. For example, the anharmonic contribution is not taken into 
account, and low-frequency modes that lead to large displacements are not treated accurately in the harmonic limit; therefore, this calculation of the entropy is to be considered a crude estimate only. Moreover, the computations for the estimation of the entropy may be the slowest step in the process. Before normal-mode analysis, the structures should be fully minimized to achieve very low RMS of the energy gradient In the current work, after conjugate gradient minimization, the NewtonRaphson technique was used. We must point out that the Newton-Raphson minimization is very time-consuming. The huge computational effort of the entropy calculations will greatly limit the practical applications of MM/PBSA in drug design. Another issue we must consider is that when the inhibitors associate with MMP-2 the water molecules near the $\mathrm{P}_{1}$ ' groups and in the S1' cavity will be rearranged. The rearrangement of the water molecules will lead to an entropy change, but in our calculations, the entropy of the rearrangnment of the water molecules is really not considered. Therefore, the development of efficient approaches to represent the entropic effects precisely and quickly may be one of the most important tasks in the further work.

\section{Conclusion}

The binding of hydroxamates to MMP-2 has been studied by means of MM/PBSA in conjunction with normal-mode analysis. This technique not only can reliably predict the relative ranking of ligands but also can successfully reproduce the experimental free energy of binding with accepted errors. It is very encouraging that the MM/PBSA technique does not need any empirical parameters, and MM/PBSA may become an attractive tool in free energy calculations.

As a comparison, the free energies of binding between hydroxamates and MMP-2 are also evaluated using LIE. The three-parameter LIE model with a constant term is proven to possess the best predictive ability. The average unsigned error for this fit between the predicted and experimental binding free energies is $0.58 \mathrm{kcal} / \mathrm{mol}$. In the current work, the potentials of the catalytic zinc center were represented by a nonbonded model, whereas in our previous work, the bonded model was used. ${ }^{55}$ Using those two different potential models, we found that the structures of the catalytic zinc bear some differences, but the obtained LIE models are of similar statistical significance. The bonded or nonbonded models may not be completely correct. We believe that whether one uses the bonded model or the nonbonded model the errors in the energetic or conformational assessment of the interaction between zinc and the ligand should be constant. Thus, the systematic error caused by the potential of zinc may be reduced in LIE fittings.

Although in the current work MM/PBSA has successfully reproduced the free energy of bindings between eight hydroxamates with MMP-2, MM/PBSA still needs to be investigated further to reduce the computational effort and improve the predictability, thereby enabling a larger set of ligands to be ranked precisely. Among the stages of free-energy calculations in MM/PBSA, the entropy estimation should be given more attention. First, the calculated entropy still has a relatively large uncertainty. Second, the stage of entropy estimation is very timeconsuming. It is necessary for us to try to apply other methods to estimate entropy, such as covariance-matrix methods or empirical methods. ${ }^{59}$ Moreover, the PB continuum calculation is also one of the more time-consuming steps in our approach, and it may be replaced by a time-saving method such as the generalized Born (GB) solvent model ${ }^{60}$ or the solvation model based on the solvent-accessible surface area (WSAS) ${ }^{61} \mathrm{Re}$ - cently, we used the WSAS developed in our group to predict the relative binding free energies of four binding modes of EGFR/quinazoline. The most favorable binding mode identified by MM-PBSA could also be correctly recognized by MMWSAS. The relative solvation free energies calculated by WSAS show obvious correlation with those calculated by PBSA ( $r=$ 0.88 ). We believe that the WSAS model is somewhat meaningful if one is interested only in the relative solvation free energy. Moreover, WSAS calculations are much faster than PBSA calculations. We believe that the MM/WSAS calculations may be very promising in estimating the binding free energies of multiple ligands in a database.

Acknowledgment. We are particularly grateful to Professor P. A. Kollman of UCSF for providing us with the AMBER 6.0 molecular simulation package and the newest Amber force field parameters. We also thank Dr. J. M. Wang of UCSF for helpful discussions of the methods concerning MD simulations and freeenergy calculations. This project is supported by the National Natural Science Foundation of China (NSFC 29992590-2 and 29873003).

Supporting Information Available: The inhibitors and the catalytic zinc cation that we studied are defined as new residue types in AMBER. The coordinates and the partial charges from ESP fitting calculations for these new residues are saved as AMBER database files. The new parameters developed by Wang et al. are listed in Table 5. All these materials can be obtained from us upon request.

\section{References and Notes}

(1) Jorgensen, W. L. Acc. Chem. Res. 1989, 22, 184-189.

(2) Straatsma, T. P.; MaCammon, J. A. Annu. Rev. Phys. Chem. 1992, 43, 407-435.

(3) Kollman, P. Chem. Rev. 1993, 93, 2395-2417.

(4) Novotny, J.; Bruccoleri, R. E.; Saul, F. A. Biochemistry 1989, 28, 4735-4749.

(5) Still, W. C.; Tempczyk, A.; Hawley, R. C.; Hendrickson. T. J. Am. Chem. Soc. 1990, 112, 6127-6129.

(6) Williams, D. H.; Cox, J. P. L.; Doig, A. J.; Gardner, M.; Gerhard, U.; Kaye, P. T.; Lal, A. R.; Nicholls, I. A.; Salter, C. J.; Mitchell, R. C. J. Am. Chem. Soc. 1991, 113, 7020-7030.

(7) Searle, M. S.; Williams, D. H.; Gerhard, U. J. Am. Chem. Soc. 1992, 114, 10690-10697.

(8) Horton, N.; Lewis, M. Protein Sci. 1992, 1, 169-181. 1684 .

(9) Bohacek, R. S.; McMartin, C. J. Med. Chem. 1992, 35, 1671-

(10) Krystek, S.; Stouch, T.; Novotny, J. J. Mol. Biol. 1993, 234, 661679.

(11) Böhm, H. J. J. Comput.-Aided Mol. Des. 1994, 8, 243-256.

(12) Head, R. D.; Smythe, M. L.; Oprea, T. I.; Waller, C. L.; Greene, S. M.; Marshall, G. R. J. Am. Chem. Soc. 1996, 118, 3959-3969.

(13) Matthew, D. E.; Christopher, W. M.; Timoghy, R. A.; Gaia, V. P.; Roger, P. M. J. Comput.-Aided Mol. Des. 1997, 11, 425.

(14) Hou, T. J.; Wang, J. M.; Chen L. R.; Xu, X. J. Protein Eng. 1999, $12,639-647$.

(15) Åqvist, J.; Medina, C.; Samuelsson, J. E. Protein Eng. 1994, 7, $385-391$.

(16) Åqvist, J.; Mowbray, S. M. J. Biol. Chem. 1995, 270, 9978-9981.

(17) Hansson, T.; Åqvist, J. Protein Eng. 1995, 8, 1137-1144.

(18) Åqvist, J.; Hansson, T. J. Phys. Chem. 1996, 100, 9512-9521.

(19) Hansson, T.; Marelius, J.; Åqvist, J. J. Comput.-Aided Mol. Des.

1998, 12, 27-35.

(20) Wang, W.; Kollman, P. A. J. Mol. Biol. 2000, 303, 567-582.

(21) Donini, O. A. T.; Kollman, P. A. J. Med. Chem. 2000, 43, 41804188.

(22) Kuhn, B.; Kollman, P. A. J. Med. Chem. 2000, 43, 3786-3791.

(23) Srinvasan, J.; Cheatham, T. E.; Cieplak, P.; Kollman, P. A.; Case, D. A. J. Am. Chem. Soc. 1998, 120, 9401-9509.

(24) Kollman, P. A.; Massova, I.; Reyes, C.; Kuhn, B.; Huo, S. H.; Chong, L.; Lee, M.; Lee, T.; Duan, Y.; Wang, W.; Donini, O.; Cieplak, P.; Srinivasan, J.; Case, D. A.; Cheatham, T. E., III Acc. Chem. Res. 2000, 33, $889-897$.

(25) Woessner, J. F. FASEB J. 1991, 5, 2145-2154. 
(26) Nagase, H.; Fields, G. B. Biopolymers 1996, 40, 399-416.

(27) Bottomley, K. M. K.; Bradshaw, D.; Nixon, J. S. Metalloproteinases as Targets for Antiinflammatory Drugs; Birkhäuser Verlag: Switzerland, 1999.

(28) Stetler-Stevenson, W. G.; Aznavoorian, S.; Liotta, L. A. Annu. Rev. Cell. Biol. 1993, 9, 541-573.

(29) Beckett, R. P.; Davidson, A. H.; Drummond, A. H. Drug Discovery Today 1996, 1, 16-26.

(30) Morgunova, E.; Tuuttila, A.; Bergmann, U.; Isupov, M.; Lindqvist, Y.; Schneider, G.; Tryggvason, K. Science (Washington, D.C.) 1999, 284, $1667-1670$.

(31) Whittaker, M.; Floyd, C. D.; Brown, P.; Gearing, A. J. H. Chem. Rev. 1999, 99, 2735-2776.

(32) Porter, J. R.; Beeley, N. R. A.; Boyce, B. A.; Mason, B.; Millican, A.; Millar, K.; Leonard, J.; Morphy, J. R.; O'Connell, J. P. Bioorg. Med. Chem. Lett. 1994, 4, 2741-2746.

(33) Broadhurst, M. J.; Brown, P. A.; Lawton, G.; Ballantyne, N.; Borkakoti, N.; Bottomley, K. M. K.; Cooper, M. I.; Eatherton, A. J.; Kilford, I. R.; Malsher, P. J.; Nixon, J. S.; Lewis, E. J.; Sutton, B. M.; Johnson, W. H. Bioorg. Med. Chem. Lett. 1997, 7, 2299-2302.

(34) Alpegiani, M.; Bissolino, P.; Absate, F.; Perrone, E.; Corigli, R.; Jabes, D. Chem. Abstr. 1999, 130, 139360

(35) Case, D. A.; Pearlman, D. A.; Caldwell, J. W.; Cheatham, T. E., III; Ross, W. S.; Simmerling, C. L.; Darden, T. A.; Merz, K. M.; Stanton, R. V.; Cheng, A. L.; Vincent, J. J.; Crowley, M.; Tsui, V.; Radmer, R. J.; Duan, Y.; Pitera, J.; Massova, I.; Seibel, G. L.; Singh, U. C.; Weiner, P. K.; Kollman P. A. AMBER 6; University of California: San Francisco, 1999.

(36) MOPAC 7.0 User's Guide; Quantum Chemistry Program Exchange (QCPE), Indiana University: Bloomington, IN, 1993.

(37) Gaussian 98 User's Guide; Gaussian, Inc.: Pittsburgh, PA, 1998.

(38) InsightII User's Guide; Molecular Simulations Inc.: San Diego, CA, 1999.

(39) SYBYL, version 6.5; Tripos Associates: St. Louis, MO, 1999.

(40) Natchus, M. G.; Cheng, M. Y.; Wahl, C. T. Bioorg. Med. Chem. Lett. 1998, 8, 2077-2080.

(41) Hoops, S. C.; Anderson, K. W.; Merz, K. M., Jr. J. Am. Chem. Soc. 1991, 113, 8262-8270.
(42) Åqvist, J. J. Phys. Chem. 1990, 94, 8021-8024.

(43) Wang, J. M.; Cieplak, P.; Kollman, P. A. J. Comput. Chem. 2000, 21, 1049-1074.

(44) Cornell, W. D.; Cieplak, P.; Bayly, C. I.; Gould, I. R.; Merz, K. M.; Ferguson, D. M.; Spellmeyer, D. C.; Fox, T.; Caldwell, J. W.; Kollman, P. A. J. Am. Chem. Soc. 1995, 117, 5179-5197.

(45) Petersson, G. A.; Bennett, A.; Tensfeldt, T. G.; Al-Laham, M. A.; Shirley, W. A.; Mantzaris, J. J. Chem. Phys. 1988, 89, 2193-3204.

(46) Cieplak, P.; Cornell, W. D.; Bayly, C.; Kollman, P. A. J. Comput. Chem. 1994, 16, 1357-1377.

(47) Jorgensen, W. L.; Chandrasekhar, J.; Madura, J.; Impey, R. W.; Klein, M. L. J. Chem. Phys. 1983, 79, 926-935.

(48) Gilson, M. K.; Sharp, K. A.; Honig, B. H. J. Comput. Chem. 1988, 9, 327-335. 1988.

(50) Sanner, M. F.; Olson, A. F.; Spehner, J. C. Biopolymers 1996, 38, $305-320$.

(51) Hou, T. J.; Wang, J. M.; Li, Y. Y.; Xu, X. J. Chin. Chem. Lett. 1998, 9, 651-654.

(52) Hou, T. J.; Wang, J. M.; Xu, X. J. Chemometr. Intell. Lab. 1999, $45,303-310$

(53) Hou, T. J.; Wang, J. M.; Liao, N.; Xu, X. J. J. Chem. Inf. Comput Sci. 1999, 39, 775-781.

(54) Toba, S.; Damodaran, K. V.; Merz, K. M., Jr. J. Med. Chem. 1999 $42,1225-1234$

(55) Hou, T. J.; Zhang, W.; Xu, X. J. J. Phys. Chem. 2001, 105, 53045315 .

(56) Paulsen, M. D.; Ornstein, R. L. Protein Eng. 1996, 9, 567-571.

(57) Wang, J.; Dixon, R.; Kollman, P. A. Proteins: Struct., Funct. Genet. 1999, 34, 69-81.

(58) Jones-Hertzog, D. K.; Jorgensen, W. L. J. Med. Chem. 1997, 40, $1539-1549$.

(59) Schlitter, J. Chem. Phys. Lett. 1993, 215, 617-621.

(60) Hawkins, G. D.; Cramer, C. J.; Truhlar, D. G. J. Phys. Chem. 1996, 100, 19824-19839.

(61) Hou, T. J.; Qiao, X. B.; Zhang, W.; Xu, X. J. J. Phys. Chem. B, in press. 